\title{
KNOWLEDGE OF REPRODUCTIVE HEALTH SOCIAL AWARENESS
}

\author{
Haque $\mathrm{DM}^{1}$
}

In Bangladesh, of the 32 million adolescent, $51 \%$ are girls. Half of them aged 15-19 years are married. Seventy percent of the pregnant girls under age 20 receive no antenatal care while $97 \%$ of births occur at home. They have lack of knowledge about their reproductive health and poor access to health service. Inevitably both married and unmarried adolescent girls are at risk for various reproductive health problems including unwanted pregnancies, risk associated with early pregnancy and sexually transmitted infections (STIs)/HIV/AIDS ${ }^{1}$.

Adolescent is a very important segment of life while a child undergoes biological transformations. World Health Organization (WHO) has defined adolescence as the age group of 10-19 years. The term adolescence literally means to emerge, to achieve identity. One fifth of the population of the world is between 10-19 years old. In Bangladesh, adolescents are about $23 \%$ of the total population ${ }^{2}$. Adolescence is a cross road in the development of life through which a child gradually walks to reach the maturity of adults ${ }^{3}$. During this important period, a child undergoes biological transition, which is characterized by puberty related changes in physical appearance and the attainment of reproductive capability.

Reproductive health need includes needs for reproductive health care, family planning, HIV/AIDS information, safer sex, unwanted pregnancy, early pregnancy, sexually transmitted diseases (STDs), safe abortion and safe motherhood. For many years, the health of adolescents has been neglected as they are less vulnerable to disease than young children or the very old $^{4}$. Specific sources of information and contraceptive advice are rarely available or accessible to them. In Bangladesh, there is poor knowledge of sexual and reproductive health among adolescents. Furthermore, what is known is often incorrect and derived through communication with friends who are equally unknowledgeable ${ }^{5,6}$. Illness relating to sexual and reproductive health may receive inadequate attention as there problems are shrouded in a culture of silence, embarrassment and shame ${ }^{7}$.

The unmet need of adolescent for reproductive health information and service is huge and diverse both in terms of quality as well as quantity. The psychological and social behavior play pivotal role that include shyness of the adolescent to discuss the reproductive health issue, keeping reproductive health problem secret, traditional values, norms and myths, ignorance about sexuality and parents/guardians and elderly people (who acts as a gate keeper) who are uninformed about reproductive health need $^{8}$.

Before the onset of sexual activity most girls meet health needs within the context of the family, relying heavily on mother for health care and advice. Many new needs and concerns emerge at sexual debut. Key factor modulating girls' ability to address their health needs and concern include the strategy of selecting disclosure of information perceived to be harmful to close family relationship on threaten privacy, the desire for personalized care modeled on the emotional and physical care received from mother. Core values shaping these processes including privacy, a close relationship with mother and a perception of sexual activity are dangerous. Various studies revealed that no girl was able to meet her specific reproductive health need within the mother daughter relationship ${ }^{8}$.

Mothers want to protect their girls against early sexual activity, pregnancy and STI and believe that they are responsible for dealing with the results of such problem. Many mothers believe that a daughter's private visit to doctor would promote risky behavior and challenge the mother daughter bond. Mother always tries to help their daughter to receive gynecological care but only after the girls become sexually active. Mother's awareness of sexual activity is low and girls always work hard to keep their sexual status private.

This issue of JAFMC highlights the role of mothers' knowledge on reproductive health needs of their adolescent girls. Here 118 respondents were interviewed to assess their knowledge for 10 related questions pertaining to mothers' knowledge about the reproductive health needs of their adolescent girls. The study was conducted at purposively selected schools of Dhaka city, hence it may not represent total population. Study findings showed that the respondents have limited

1. Brig Gen Dewan Masudul Haque MBBS, DPH, M Phil, Professor and Head, Department of Community Medicine, AFMC 
knowledge about reproductive needs and have limited access to accurate information on service, though all mother were educated and represented affluent urban society. The relationship between education, occupation and source of information with knowledge were found statistically significant.

Mothers are very intimate with their girls in every aspect of life. Mothers are the best educators too. On the other hand, adolescent girls conceal their reproductive needs from mother. Intervention to be made in health system to educate mothers about reproductive needs of their adolescent children and to create or inculcate such relationship, so that, their adolescent girls love to share their reproductive needs with their mothers. In Bangladesh, very little is known about reproductive health needs of adolescent. Adolescent do not have access to correct information about reproduction, fertility regulation and their reproductive health needs are largely ignored by current program activities. Major source of information of adolescent's physical change and reproductive need is mother. But, often, she is unable to meet those queries ${ }^{9}$.

\section{Refernces}

1. Bhuiya I. Strategies to improve reproductive health of adolescent girls in Bangladesh experience from a project. The 131st Annual Meeting of APHA; 2003 November 15-19, Dhaka: 2003.

2. Agampodi SB, Agampodi TC, Piyaseeli UKD. Adolescent perception of reproductive health care service in Sri Lanka. BMC Health Service Research 2008 May 3; 8:98 (1-8).

3. Shadhna G, Achala S, Awareness about reproductive and adolescent charges among school girls of different socio-economic status. J obstet Gynecol India 2006, 56(4): 324-328.

4. Ackard DM, Neomark-Sztainer D. Health care information source for adolescent: Age and gender difference on use, concerns and needs. Journal of Adolescent Health 2001; 29:170-176.

5. Mitra SN, Al-Sabir, Saha I, Kumar S. Bangladesh demographic and health survey 1999-2000, Dhaka: National Institute of Population Research and Training; 2001.

6. Nahar Q, T uhon I Houvras R, Gazi M, Reza, Huq NL, Khula B. Reproductive health needs of adolescents in Bangladesh : a study report. Dhaka: International Center for Diarrhoeal Diseases and Research Bangladesh(ICDDRB); 1999. p.130.

7. Huq NL, Haseen F, Quaiyum MA, Nahar Q, Larson CP, Strategies to improve reproductive Health Service for adolescents in Bangladesh : A worksite study. Dhaka: ICDDRB workings paper; 2005. p.165

8. McKee DM, Karasz A, Catherine M. Weber. Health Care Seeking Among Urban Minority Adolescent Girls: The Crisis at Sexual Debut. Ann Fam Med. 2004 November; 2(6): 549-554.

9. Rob UL, Bhuiya LR, Yusuf N. Improving adolescents' reproductive health in Bangladesh research update no-2. Dhaka: Bangladesh population council 2002 